Revista de la Escuela de Ciencias de la Educación, año 13, nro. 12, vol. 1, enero a junio de 2017. PáglNAS 175-196. ISSN 1851-6297 - ISSN 2362-3349 (EN LiNEA) DIFERENCIACIÓN ESCOLAR Y EXPERIENCIAS PEdagógicas en espacios locales: el caso de las escuelas secundarias de Rosario. Jorgelina Silvia Sassera

\title{
DIFERENCIACIÓN ESCOLAR Y EXPERIENCIAS PEDAGÓGICAS EN ESPACIOS LOCALES: EL CASO DE LAS ESCUELAS SECUNDARIAS DE ROSARIO
}

\author{
Por Jorgelina S. Sassera* \\ Universidad de Buenos Aires - Argentina \\ jsassera@filo.uba.ar
}

Recibido: 20/09/2016 Aceptado: 22/10/2016

\section{Resumen}

En el marco de sistemas educativos segmentados en los que se consolida la diferenciación institucional en torno a las desigualdades en los recursos materiales y humanos, la localización y la accesibilidad a las escuelas, cabe la pregunta sobre los aprendizajes a los cuales los estudiantes acceden. Como un modo de aproximación a esta cuestión se aborda la realización de experiencias pedagógicas entendidas como una forma de transmisión mediante la experiencia directa de contenidos, actitudes y valores. El desarrollo de estas experiencias en Rosario ocurre en escuelas con características diferentes que en algunos casos pueden conducir a aprendizajes desiguales. En este sentido, el artículo da cuenta de la existencia de tres grupos de escuelas secundarias en cuanto a los recursos edilicios de los que disponen y a las que asisten

* Doctora de la Universidad de Buenos Aires en Ciencias de la Educación. Magister en Ciencias Sociales con Orientación en Educación (FLACSO Argentina). Lic. en Sociología (UBA). Investigadora del Programa Educación, Economía y Trabajo (PEET), Instituto de Investigaciones en Ciencias de la Educación, Facultad de Filosofía y Letras (UBA). Ha sido Becaria Doctoral de CONICET con sede en el Programa de Educación, Economía y Trabajo del Instituto de Investigaciones en Ciencias de la Educación de la Facultad de Filosofía y Letras de la Universidad de Buenos Aires 
Revista de la Escuela de Ciencias de la Educación, año 13, nro. 12, vol. 1, enero a junio de 2017. PáglNAS 175-196. ISSN 1851-6297 - ISSN 2362-3349 (EN LINEA) DIFERENCIACIÓN ESCOLAR Y EXPERIENCIAS PEdagógicas en espacios locales: el caso de las escuelas secundarias de Rosario. Jorgelina Silvia Sassera

estudiantes con distinto origen socioeconómico. El artículo relata las experiencias pedagógicas realizadas en cada grupo de escuelas buscando esclarecer qué factores intervienen en la posibilidad de desarrollo de las mismas y en las condiciones de participación de los estudiantes.

Palabra Clave: Educación secundaria - Rosario - Segmentación socio-educativa - Diferenciación - Experiencias pedagógicas

\begin{abstract}
Within the framework of segmented educational systems in which the institutional differentiation is growing around the inequalities in the material and human resources, the localization and accessibility to schools, it is relevant to ask about the access to learning. A way to approach this question is though the pedagogic experiences understood as a mean of transmission of contents, attitudes and values. The development of these experiences in Rosario happens on schools with different characteristics that in some cases might lead to unequal learning. The article sets the existence of three groups of secondary schools referring to the available buildings resources and the different socio-economical origin of the students. The article describes the pedagogic experiences that take place in each group of schools seeking to establish the factors that intervene in the possibilities of their development and in the conditions for the participation of the students.
\end{abstract}

Keywords: Secondary education - Rosario - Socio-educative segmentation - Differentiation - Pedagogic experiences

La diferenciación de las escuelas secundarias se plantea como un problema de estudio en el marco de sistemas educativos segmentados que no sólo limitan el acceso a la educación sino que favorecen condiciones desiguales para el desarrollo de los aprendizajes de los adolescentes. La segmentación educativa crea condiciones materiales distintas (e incluso desiguales) entre las escuelas que se distinguen en cuanto a las condiciones edilicias, las características de los docentes y la composición socio-económica de los estudiantes. Asimismo esta diferenciación se relaciona con la localización de las escuelas, aquellas instituciones localizadas en los espacios con mayores desventajas sufren asimismo las mayores ausencias de recursos.

Una forma de aproximación al problema de las condiciones desiguales y los aprendizajes a los que acceden los estudiantes consiste en el estudio de las experiencias pedagógicas (cuyos propósitos son formar a los estudiantes en determinados contenidos, valores y actitudes) y que se realizan en instituciones concretas a su vez insertas en espacios locales precisos que pueden favorecer o limitar su desarrollo. 
Revista de la Escuela de Ciencias de la Educación, año 13, nRo. 12, vol. 1, enero a junio de 2017. PáglNAS 175-196. ISSN 1851-6297 - ISSN 2362-3349 (EN LiNEA) DIFERENCIACIÓN ESCOLAR Y EXPERIENCIAS PEdagógicas en espacios locales: el caso de las escuelas secundarias de Rosario. Jorgelina Silvia Sassera

El análisis de las experiencias pedagógicas parte desde un marco teórico y conceptual que enmarca a la educación secundaria en la diferenciación y segmentación en espacios locales. Este artículo presenta los resultados de una investigación en curso (1) que abarca a localidades de la provincia de Buenos Aires (Campana, Zárate, Florencio Varela y Berazategui) y de Santa Fe (Villa Constitución, Empalme y Rosario), aunque en esta oportunidad se hace hincapié en la ciudad de Rosario.

La primera parte el artículo presenta brevemente el abordaje teórico- conceptual sobre la diferenciación, la segmentación y las experiencias pedagógicas; la segunda parte las dimensiones institucionales de la diferenciación escolar y segmentación socio-urbana en Rosario. Luego el artículo presenta la existencia de tres grupos de escuelas diferentes bajo la noción de "diferenciación difusa" pues las diferencias entre escuelas se presentan de manera gradual y matizada; finalmente el artículo vincula la realización de experiencias pedagógicas específicas con las características de cada circuito y las condiciones de acceso a las mismas por parte de los estudiantes.

\section{Segmentación educativa y espacial en la educación secundaria: el sentido de las experiencias pedagógicas de los adolescentes}

El tratamiento de la segmentación educativa y espacial requiere tres ejes de análisis principales: la dimensión espacial expresada en los espacios locales concretos; la desigualdad educativa y la segmentación del sistema educativo; y es en este contexto de interpretación que el estudio de las experiencias pedagógicas de los adolescentes de las escuelas secundarias cobra sentido pues se posibilita su compresión en función de los circuitos institucionales y las características de la accesibilidad a las mismas.

La dimensión espacial manifestada en la ciudad, se configura como arquitectura moldeada por las tendencias macro-sociales y en la que impactan los procesos internacionales de la globalización, los avances tecnológicos y las mutaciones del nivel estatal - nacional. Pero el espacio de lo local no es uniforme ni estático, pues en él se manifiestan las diferencias y las desigualdades que se materializan en formas de segregación entre clases sociales y estratos o fracciones de clases sociales. En el espacio (físico y social) un lugar no es equivalente a otro, pues existen apropiaciones desiguales de los bienes, servicios públicos y colectivos y de los capitales culturales y sociales desigualmente distribuidos.

En los espacios locales cobran forma los procesos de la desigualdad educativa y en las localidades se pueden esclarecer los factores y mecanismos que intervienen. La segmentación educativa expresa múltiples diferencias y desigualdades, y en nuestro país se ha caracterizado no sólo por una diferenciación entre instituciones privadas y públicas, sino especialmente por la desigual prestación de los establecimientos públicos resultando en aprendi- 
Revista de la Escuela de Ciencias de la Educación, año 13, nro. 12, vol. 1, enero a junio de 2017. PáglNAS 175-196. ISSN 1851-6297 - ISSN 2362-3349 (EN LINEA) DIFERENCIACIÓN ESCOLAR Y EXPERIENCIAS PEdagógicas en espacios locales: el caso de las escuelas secundarias de Rosario. Jorgelina Silvia Sassera

zajes diferentes para grupos sociales diferentes. Esta desigualdad ocurre en la dotación de recursos materiales y humanos, las características edilicias, las capacidades de gestión y de desempeño técnico - pedagógico de los docentes, la accesibilidad a las propias instituciones y a las posibilidades de las instituciones de articularse o vincularse con otras instituciones educativas, gubernamentales, sociales y productivas. Además de estos factores, las escuelas públicas se han distinguido en prestigio y métodos de selección explícitos e implícitos para la atracción o rechazo de distintos sectores sociales (Braslavsky, 1994).

La desigualdad al interior de un sistema segmentado se sustenta en la existencia de circuitos, es decir grupos de instituciones de un mismo nivel educativo con características similares que se dirigen a un grupo de población.

Estas brechas entre grupos de escuelas son tributarias de muchos factores y si bien formalmente, en nuestro país, no hay barreras explícitas que impidan el pasaje de los estudiantes entre instituciones, las prácticas de los actores por mantener ciertas posiciones de privilegio o por el contrario, de salir de posiciones desventajosas, pueden profundizar las desigualdades. Así, una norma que administrativamente busca regular el funcionamiento cotidiano de una institución, por ejemplo en el momento de inscripción o en las maneras de distribución de vacantes, puede dar lugar a prácticas selectivas debido a las interpretaciones por parte de los actores encargados de aplicarlas efectivamente (Sassera, 2013a).

Además, la continuación de la selección de los estudiantes a lo largo de la escolarización y los modos de encausamiento de "tipos de estudiantes para escuelas o instituciones acordes a ellos" da cuenta de la búsqueda por el mantenimiento lógicas de diferenciación que rápidamente trocan en prácticas que generan desigualdad, ya que terminan homogenizando las poblaciones estudiantiles. Otro factor que incide en la conformación de grupos de escuelas y que afecta el acceso a la educación son las barreras, cuya existencia promueven la discriminación de la población que se ve limitada o impedida de acceder a estos servicios, profundizando aún más las malas condiciones materiales y de vida (Riquelme, 1989).

El abordaje teórico define conceptualmente a la noción de experiencia pedagógica, considerándola como una categoría imprecisa y ambivalente. En primera instancia, las experiencias -en plural porque difícilmente son únicastienen dos dimensiones: una apunta al flujo vital y de emociones, a la interiorización de una externalidad que cambiaría al sujeto. La otra dimensión alude a los marcos institucionales, a las estrategias y la resolución de problemas.

Las experiencias se desarrollan en marcos institucionales que las sustentan y regulan. Por ejemplo, alumnos y docentes buscan dar coherencia a sus prácticas en el marco de la escuela para afrontar problemáticas concretas, por ejemplo cómo lograr transmitir a los estudiantes determinadas habilidades, 
Revista de la Escuela de Ciencias de la Educación, año 13, nRo. 12, vol. 1, enero a junio de 2017. PáglNAS 175-196. ISSN 1851-6297 - ISSN 2362-3349 (EN LiNEA) DIFERENCIACIÓN ESCOLAR Y EXPERIENCIAS PEdagógicas en espacios locales: el caso de las escuelas secundarias de Rosario. Jorgelina Silvia Sassera

valores y actitudes que no estarían contempladas en las prácticas escolares cotidianas. Entonces, siguiendo la propuesta de Dubet (2011), la noción de experiencia da cuenta de las estrategias de los actores para resolver problemas en marcos institucionales siguiendo determinados propósitos y objetivos propuestos por estos actores; también implican los sentidos atribuidos por estas experiencias por parte de los sujetos que las desarrollan y transitan.

En las formas en que se justifican y plantean, en las experiencias pedagógicas se pueden percibir las huellas del pensamiento de Dewey y de la educación progresiva. Las experiencias pedagógicas surgen de dos maneras: en oposición o complementariedad con las prácticas áulicas cotidianas. Ya sea porque el currículum es inadecuado, o la relación docente - estudiante es percibida como rígida o porque los libros no son suficientes y no despiertan el interés en los estudiantes; el aprendizaje mediante la experiencia supone la motivación, la creatividad y la vitalidad presente para el logro de la adquisición de saberes, habilidades y actitudes que de otra forma no serían "despertadas" en los estudiantes.

Las experiencias pedagógicas pueden ser consideradas como una modalidad de práctica pedagógica (2), en la que se pone el eje en el aprendizaje mediante la experiencia para la transmisión de contenidos curriculares, de valores, actitudes y reglas. El aprendizaje mediante la experiencia es fomentado desde el sector educativo, aunque también desde otros ámbitos, y es formulado como una crítica al aprendizaje teórico y a los dispositivos "tradicionales" tales como la lección magistral o la exposición del docente. Justamente, se fomenta la realización práctica y la generación de experiencias subjetivas que redundarían en mejores aprendizajes y en cambios subjetivos en los estudiantes; se trata de una formación que supera al "aprender haciendo" para acentuar el "aprender ser".

\section{Acerca de la educación secundaria de Rosario: dimensiones institu- cionales y segmentación urbana}

Rosario es una de las ciudades más relevantes del país y conforma una amplia área metropolitana. Entre las múltiples caracterizaciones que podrían realizarse sobre esta ciudad y su área metropolitana, interesa destacar aquellos diagnósticos que dan cuenta de las desigualdades materializadas en distribuciones desiguales de servicios públicos, las concentraciones de bajos niveles educativos, subempleo y redes sociales débiles en áreas urbanas distinguidas por sus situaciones de violencia en contraposición con zonas que concentran la infraestructura y equipamiento (Herzer, 2005).

Las áreas más desfavorecidas se insertan en los intersticios de la trama urbana (por ejemplo a los costados de la red ferroviaria) o en la periferia demarcada con la autopista Circunvalación (3). La distribución de las condiciones desiguales es heterogénea, pues existen disparidades entre los distritos 
Revista de la Escuela de Ciencias de la Educación, año 13, nro. 12, vol. 1, enero a junio de 2017. PágiNAS 175-196. ISSN 1851-6297 - ISSN 2362-3349 (EN LINEA) DIFERENCIACIÓN ESCOLAR Y EXPERIENCIAS PEdagógicas en espacios locales: el caso de las escuelas secundarias de Rosario. Jorgelina Silvia Sassera

y los barrios que los componen. La ciudad presenta un patrón radiocéntrico (Herzer, 2005; Sassera 2014) y las zonas más desfavorecidas son el noroeste y suroeste, aunque existen situaciones de asentamientos insertas en la trama urbana céntrica que reclaman urbanización y regularización de la calidad de los servicios públicos. Un indicador concreto de estas situaciones de desigualdad es la proporción de adolescentes de entre 12 y 18 años que trabaja $(19,18 \%$ según datos del Censo 2010$)$. Esto significa que las familias no pueden garantizar el sustento de los adolescentes, por otra parte el trabajo de un adolescente puede afectar negativamente su escolarización.

Las características mencionadas de la ciudad de Rosario permiten dar cuenta de la situación social del espacio local estudiado. Es en los espacios locales concretos donde toman forma las desigualdades educativas y en los que se pueden dar cuenta de los procesos concretos que intervienen en la diferenciación institucional y la segmentación educativa.

El estudio de las instituciones educativas consideradas en la investigación, se realizó buscando integrar las diversas dimensiones que producirían diferenciación entre establecimientos escolares. Para comprender estos procesos, se consideraron no sólo los aspectos materiales, sino también aquellos referidos con las características de los docentes, los estilos (y posibilidades y limitaciones) de la gestión institucional por parte del equipo directivo y las relaciones y vinculaciones de las instituciones educativas con otras de la realidad social y productiva. También se tomaron en cuenta aspectos pedagógicos, relacionados con las posibles adaptaciones curriculares que se pudieran realizar y con el desarrollo de determinadas experiencias pedagógicas.

Cuadro 1. Dimensiones de la diferenciación entre establecimientos educativos.

\begin{tabular}{|c|c|c|c|c|}
\hline \multicolumn{5}{|c|}{ Dimensiones de la diferenciación entre establecimientos educativos } \\
\hline Material & Docente & Gestión institucional & Relacional & Pedagógica \\
\hline $\begin{array}{l}\text {-Aulas suficientes } \\
\text { y disponibilidad } \\
\text { de biblioteca, } \\
\text { laboratorios, sala } \\
\text { de arte, gimnasio } \\
\text { y patio. }\end{array}$ & $\begin{array}{l}\text {-Formación } \\
\text { docente. }\end{array}$ & $\begin{array}{l}\text {-Prácticas implícitas } \\
\text { o explícitas de selec- } \\
\text { ción de la matrícula. }\end{array}$ & $\begin{array}{l}\text { - Apertura a } \\
\text { vínculos con } \\
\text { organizaciones } \\
\text { y actores ex- } \\
\text { tra- escolares. }\end{array}$ & $\begin{array}{l}\text {-Adaptacio- } \\
\text { nes curricu- } \\
\text { lares. }\end{array}$ \\
\hline $\begin{array}{l}\text {-Estado de man- } \\
\text { tenimiento e } \\
\text { higiene. }\end{array}$ & $\begin{array}{l}\text { - Experiencia } \\
\text { y antigüedad. }\end{array}$ & $\begin{array}{l}\text { - Coordinación con } \\
\text { otras instituciones } \\
\text { educativas. }\end{array}$ & $\begin{array}{l}\text { - Participación } \\
\text { en certámenes } \\
\text { y ferias esco- } \\
\text { lares. }\end{array}$ & $\begin{array}{l}\text { - Realización } \\
\text { de experien- } \\
\text { cias pedagó- } \\
\text { gicas. }\end{array}$ \\
\hline
\end{tabular}


Revista de la Escuela de Ciencias de la Educación, año 13, nRo. 12, vol. 1, enero a junio de 2017. PáglNAS 175-196. ISSN 1851-6297 - ISSN 2362-3349 (EN LiNEA) DIFERENCIACIÓN ESCOLAR Y EXPERIENCIAS PEdagógicas en espacios locales: el caso de las escuelas secundarias de Rosario. Jorgelina Silvia Sassera

\begin{tabular}{|c|c|c|c|c|}
\hline $\begin{array}{l}\text {-Disponibilidad } \\
\text { de Equipamiento } \\
\text { mínimo, de equi- } \\
\text { pamiento relacio- } \\
\text { nado con las nue- } \\
\text { vas tecnologías: y } \\
\text { de equipamiento } \\
\text { específico a la } \\
\text { orientación y mo- } \\
\text { dalidad. }\end{array}$ & $\begin{array}{l}\text { - Acto público } \\
\text { y asignación } \\
\text { de los do- } \\
\text { centes para } \\
\text { la cobertura } \\
\text { de cargos y } \\
\text { suplencias. }\end{array}$ & $\begin{array}{l}\text { - Gestión de subsi- } \\
\text { dios, donaciones y } \\
\text { fuentes alternativas } \\
\text { de financiamiento. }\end{array}$ & $\begin{array}{l}\text { - Gestión de } \\
\text { visitas y excur- } \\
\text { siones. }\end{array}$ & \\
\hline \multicolumn{5}{|c|}{ Dimensión espacial: segmentación urbana y segregación socio- residencial } \\
\hline $\begin{array}{l}\text { - Localización } \\
\text { de la institución } \\
\text { (barrio, distrito, } \\
\text { ámbito urbano o } \\
\text { rural). }\end{array}$ & $\begin{array}{l}\text { - Caracterís- } \\
\text { ticas socio- } \\
\text { económicas } \\
\text { de la pobla- } \\
\text { ción. }\end{array}$ & $\begin{array}{l}\text { - Distribución de los } \\
\text { servicios públicos. }\end{array}$ & - Accesibilidad. & $\begin{array}{l}\text { - Barreras } \\
\text { físicas y sim- } \\
\text { bólicas. }\end{array}$ \\
\hline
\end{tabular}

Fuente: elaboración propia. PEET-IICE-FFyL/UBA

El análisis de Rosario en cuanto espacio local concreto con sus heterogeneidades urbanas y sociales da el marco para el estudio de los modos de aplicación de las políticas educativas y la fisonomía específica que adoptan las instituciones y las prácticas de los actores en el campo de la educación. A tal fin, se ha seleccionado para el análisis a escuelas secundarias orientadas (EESO) y escuelas técnicas (ET) de gestión estatal con diversa distribución en el espacio rosarino.

Una matriz universalista de la educación supondría que todos los establecimientos son semejantes en sus características edilicias, sus recursos, docentes y condiciones de enseñanza. Sin embargo, se ha producido una diferenciación creciente entre escuelas, que ha transformado las diferencias en desigualdades. Las características y mantenimiento de los edificios, la disponibilidad de espacios tales como laboratorios, bibliotecas, gimnasios y salas de arte, la experiencia y capacitación de los docentes y los recursos monetarios disponibles han sido identificados como factores que diferencian a las escuelas entre sí y que contribuyen a la generación de circuitos educativos.

Si bien la posibilidad de conocer con certeza el impacto de estas diferencias sobre el aprendizaje es elusiva, es posible suponer que inciden sobre la experiencia escolar de los estudiantes y sobre las condiciones de trabajo de los docentes. Por otra parte, cuando la diferencia se convierte en ausencia de un factor relevante, la misma pasa a convertirse en una desigualdad del cumplimiento de un derecho que debería ser para todos sin importar las condiciones materiales de los sujetos que acceden a esos derechos. La investiga- 
Revista de la Escuela de Ciencias de la Educación, año 13, nRo. 12, vol. 1, enero a junio de 2017. PáglNAS 175-196. ISSN 1851-6297 - ISSN 2362-3349 (EN LINEA) DIFERENCIACIÓN ESCOLAR Y EXPERIENCIAS PEdagógicas en espacios locales: el caso de las escuelas secundarias de Rosario. Jorgelina Silvia Sassera

ción en curso muestra evidencias de que en las localidades de Buenos Aires estudiadas coinciden la desfavorabilidad de las zonas donde se emplazan las instituciones educativas, las situaciones de pobreza y desigualdad de la población y los establecimientos educativos sin condiciones adecuadas tanto materiales como de recursos humanos.

\section{La diferenciación institucional educativa difusa en Rosario}

Las características de Rosario como ciudad, así como de las propias instituciones educativas limitan la posibilidad de dar cuenta de una marcada tendencia hacia la segmentación y la segregación educativas. En este sentido, es posible hacer referencia a una "diferenciación difusa" (Sassera, 2014), pues a diferencia de lo hallado en las otras localidades estudiadas (Sassera 2013a y b, Sassera 2014, Herger y Sassera, 2015), existe una mayor heterogeneidad en la matrícula al interior de casi todas las escuelas.

Al ser una ciudad con gran desarrollo urbano, lo cual implica extensión de los servicios públicos y un mejor funcionamiento del sistema de transporte público (a diferencia de situaciones estudiadas en localidades bonaerenses), las problemáticas de desfavorabilidad y accesibilidad a las instituciones aparecen matizadas debido a los menores quiebres y dispersión en las trazas urbanas de la ciudad. Este ha sido un factor considerado para la evaluación de las barreras físicas que podrían limitar la asistencia de los estudiantes a las instituciones educativas.

Puede afirmarse que existen condiciones de relativa similitud entre las escuelas rosarinas en cuanto a las condiciones edilicias, materiales didácticos y capacidad de gestión de los equipos directivos. Por ello, en la construcción tentativa de circuitos, se tuvo en cuenta principalmente el origen socio - económico y la situación de estudio y trabajo de los alumnos. Vale recordar los efectos negativos de la homogeneización de la matrícula:

La división social del sistema educativo transforma a las escuelas en guetos en los que sólo se encuentran los miembros de sectores sociales semejantes. En estas circunstancias, la experiencia educativa de los alumnos más desfavorecidos se ve especialmente perjudicada, ya que sus posibilidades de aprendizaje y desarrollo futuro se reducen de manera significativa (Veleda, 2012. p. 17).

La investigación muestra que en algunas escuelas de la ciudad, especialmente las del sur se registra una concentración de estudiantes con características similares que configuran poblaciones estudiantiles más homogéneas que en otras instituciones rosarinas. 
Revista de la Escuela de Ciencias de la Educación, año 13, nRo. 12, vol. 1, enero a junio de 2017. PáglNAS 175-196. ISSN 1851-6297 - ISSN 2362-3349 (EN LíNEA) DIFERENCIACIÓN ESCOLAR Y EXPERIENCIAS PEDAGógICAS EN ESPACIOS locales: EL CASO DE LAS ESCUELAS SECUNDARIAS DE Rosario. JoRgELINA Silvia SASSERA

Cuadro 2. Síntesis de las características de los grupos de escuelas. Rosario.

\begin{tabular}{|c|c|c|c|}
\hline & Barreras & $\begin{array}{l}\text { Condiciones edilicias, } \\
\text { espacios y materiales }\end{array}$ & Perfil de la matrícula \\
\hline \multicolumn{4}{|l|}{ Rosario } \\
\hline $\begin{array}{l}\text { Grupo 1. EI } \\
\text { Centro I } \\
\text { E.E.T. L } \\
\text { E.E.T P } \\
\text { E.E.T O } \\
\text { E.E.M. C }\end{array}$ & $\begin{array}{l}\text { Sin barreras } \\
\text { mencionadas. }\end{array}$ & $\begin{array}{l}\text { Edificios propios, con } \\
\text { aulas, talleres, laborato- } \\
\text { rios y bibliotecas, y otros } \\
\text { espacios como salas de } \\
\text { arte y computación. Se } \\
\text { los clasifica de muy bueno } \\
\text { a adecuados, algunos } \\
\text { en obras de ampliación. } \\
\text { Diversos materiales didác- } \\
\text { ticos y de equipamiento. } \\
\text { Diversidad de fuentes de } \\
\text { financiamiento nacionales, } \\
\text { provinciales, municipales, } \\
\text { empresas. }\end{array}$ & $\begin{array}{l}\text { Matrícula de clase } \\
\text { media, media baja con } \\
\text { padres con diversos } \\
\text { niveles educativos: } \\
\text { desde primaria com- } \\
\text { pleta hasta universi- } \\
\text { tario. Principalmente } \\
\text { asisten a la escuela } \\
\text { desde el centro, des- } \\
\text { de diversos barrios y } \\
\text { desde otros munici- } \\
\text { pios. La mayoría sólo } \\
\text { estudia, y "los menos } \\
\text { estudian y trabajan". }\end{array}$ \\
\hline $\begin{array}{l}\text { Grupo 2. EI } \\
\text { Centro II } \\
\text { E.E.T K } \\
\text { E.E.M B } \\
\text { EET M } \\
\text { E.E.T H } \\
\text { E.E.T. I } \\
\text { EESO D } \\
\text { Noroeste } \\
\text { EESO N } \\
\text { Sur } \\
\text { EESO D }\end{array}$ & $\begin{array}{l}\text { Sin barreras } \\
\text { mencionadas. }\end{array}$ & $\begin{array}{l}\text { Edificios con aulas, talle- } \\
\text { res, laboratorios y bibliote- } \\
\text { cas, aunque con necesidad } \\
\text { de mejora y ampliación. } \\
\text { Edificios compartidos. } \\
\text { Diversos materiales didác- } \\
\text { ticos y de equipamiento. } \\
\text { Diversidad de fuentes de } \\
\text { financiamiento nacionales, } \\
\text { provinciales, municipales, } \\
\text { empresas. }\end{array}$ & $\begin{array}{l}\text { Matrícula de clase } \\
\text { media, media baja } \\
\text { y trabajadora con } \\
\text { padres con nivel edu- } \\
\text { cativo predominante: } \\
\text { primaria. } \\
\text { Casos de estudian- } \\
\text { tes que trabajan en } \\
\text { changas o en tareas } \\
\text { domésticas o de cui- } \\
\text { dado. Intervenciones } \\
\text { institucionales. }\end{array}$ \\
\hline $\begin{array}{l}\text { Grupo III } \\
\text { Norte } \\
\text { E.E.M E } \\
\text { E.E.M F } \\
\text { E.E.T. R } \\
\text { Noroeste } \\
\text { E.E.TP } \\
\text { E.E.T. Q } \\
\text { Sur } \\
\text { E.E.T T } \\
\text { E.E.T S } \\
\text { E.E.T U }\end{array}$ & $\begin{array}{l}\text { - La ubicación en } \\
\text { la periferia. } \\
\text { - Calles cortadas } \\
\text { y vías de tren } \\
\text { que impiden el } \\
\text { paso. } \\
\text { - Menor cantidad } \\
\text { de transporte } \\
\text { público. } \\
\text { - Cercanía con la } \\
\text { Circunvalación. }\end{array}$ & $\begin{array}{l}\text { Edificios adecuados, pero } \\
\text { con necesidad de amplia- } \\
\text { ción. En algunos casos } \\
\text { ausencia de taller y labo- } \\
\text { ratorios. } \\
\text { Menor disponibilidad de } \\
\text { recursos financieros, prin- } \\
\text { cipalmente Plan de Mejo- } \\
\text { ras e INET. Instituciones } \\
\text { con Copa de Leche y Plan } \\
\text { Nacional Nutricional. }\end{array}$ & $\begin{array}{l}\text { - Estudiantes de clase } \\
\text { trabajadora y baja. } \\
\text { - En algunas escuelas, } \\
\text { AUH y otros subsidios } \\
\text { nacionales y provin- } \\
\text { ciales. } \\
\text { - Padres con muy bajo } \\
\text { nivel educativo. } \\
\text { - En el sur problemas } \\
\text { de retención, pues al } \\
\text { conseguir trabajo los } \\
\text { estudiantes dejan la } \\
\text { escuela. }\end{array}$ \\
\hline
\end{tabular}

Fuente: Elaboración propia. PEET-IICE-FFyL/UBA 
Revista de la Escuela de Ciencias de la Educación, año 13, nro. 12, vol. 1, enero a junio de 2017. PáglNAS 175-196. ISSN 1851-6297 - ISSN 2362-3349 (EN LINEA) DIFERENCIACIÓN ESCOLAR Y EXPERIENCIAS PEdagógicas en espacios locales: el caso de las escuelas secundarias de Rosario. Jorgelina Silvia Sassera

El estudio de los factores de diferenciación en veinte escuelas secundarias dio lugar a la construcción de tres grupos o circuitos escolares. Vale recordar que estos agrupamientos tipológicos constituyen ficciones del investigador y que no pretenden fijar esta caracterización como única o permanente. Estos grupos construidos permiten comparar las distintas condiciones edilicias, las barreras percibidas y las características de los estudiantes considerando además la localización según distrito. Así se observa que en el Grupo III que contiene a las instituciones con mayores situaciones de desventajas incluye a escuelas localizadas en el Norte, el Noroeste y en el Sur, aunque ninguna perteneciente al Centro.

Este grupo se caracteriza por la presencia de barreras específicas, tales como calles y obstáculos que impiden el paso de los estudiantes hacia las escuelas, menor cantidad y frecuencia en el transporte público y la presencia de la Autopista Circunvalación como una barrera física y simbólica que constituye un elemento de preocupación en las escuelas. Asimismo en este grupo se pudo dar cuenta de la presencia de políticas específicas de nutrición y alimentación orientadas a la población en situación desfavorable y una descripción de la composición de la matrícula con características homogéneas. Esto contrasta principalmente con las situaciones relevadas en el grupo I, cuya matrícula es caracterizada como heterogénea y en el que no se registran barreras a la accesibilidad.

Este análisis de la diferenciación escolar en cuanto a las condiciones materiales habilita el estudio de otras dimensiones de la diferenciación en los terrenos de lo pedagógico, expresado en la presencia o ausencia de experiencias pedagógicas y el acceso de los estudiantes a las mismas.

4. La relación entre diferenciación educativa y experiencias pedagógicas en las escuelas secundarias de Rosario. ¿Qué aprendizajes para los adolescentes rosarinos?

El estudio de la diferenciación entre escuelas secundarias en espacios locales concretos se ha realizado teniendo en cuenta a la dimensión de las tareas de enseñanza y aprendizaje, considerando que la desigualdad educativa ocurre no solamente en el acceso a las instituciones educativas sino que puede continuar dentro de las mismas. Como una vía de acceso a este problema, se han relevado y analizado diversas experiencias pedagógicas pudiendo identificar los objetivos y sentidos otorgados por directores, docentes y estudiantes.

Un elemento central de las experiencias estudiadas, son los objetivos implícitos y explícitos que se proponen y que actúan como un horizonte de dirección de las actividades y prácticas desarrolladas. A partir de estos propósitos, ha sido posible reconstruir tres tipos de experiencias:

- Experiencias pedagógicas orientadas al mundo del trabajo: se proponen 
Revista de la Escuela de Ciencias de la Educación, año 13, nRo. 12, vol. 1, enero a junio de 2017. PáglNAS 175-196. ISSN 1851-6297 - ISSN 2362-3349 (EN LiNEA) DIFERENCIACIÓN ESCOLAR Y EXPERIENCIAS PEdagógicas en espacios locales: el caso de las escuelas secundarias de Rosario. Jorgelina Silvia Sassera

brindar conocimientos, habilidades y, valga la redundancia, experiencia a los estudiantes. Parten del supuesto de que existe un conocimiento demandado por el mercado de trabajo y por algunos sectores productivos que la escuela no brinda y que debería hacerlo.

- Experiencias pedagógicas de formación ciudadana y de integración social y juvenil: apuntan a la transmisión de valores y actitudes relacionados con la construcción ciudadana y la política, el establecimiento de vínculos comunitarios tanto al interior del establecimiento educativo como con el afuera del mismo.

- Experiencias pedagógicas de fortalecimiento del aprendizaje: el eje es la promoción de formas de aprendizaje alternativas, pues las prácticas áulicas regulares se mostrarían como insuficientes para la incorporación de ciertos conocimientos y habilidades. También, tienen como centro el generar motivación, entusiasmo y promover la creatividad.

Al interior de estos tipos además se distinguen dispositivos específicos mediante los cuales se concretan las experiencias según las condiciones que las posibilitan. Existen diversidad de dispositivos, algunos de ellos cuentan con historia y tradición en las instituciones educativas, como lo son la exposición magistral o la lección paseo, mientras que otros son más novedosos pues recurren a las nuevas tecnologías o han surgido ad-hoc para dar respuesta a una necesidad surgida en el aula o en la institución.

La legitimidad de las experiencias ad- hoc emerge de la normativa educativa vigente, en la cual se expresa que "se trata de establecer un lazo entre las experiencias valiosas que las escuelas vienen desarrollando y las nuevas iniciativas institucionales. En este contexto también se resignifica la relación entre lo tradicional y lo innovador en las escuelas" (CFE, Res. 93/09. p. 16).

Muchas de las experiencias surgen por iniciativa propia de las instituciones, ya sea de parte del equipo directivo, los docentes o los propios estudiantes. Otras, se implementan como resultado de propuestas realizadas por distintas organizaciones externas a la escuela y su legitimación desde la institución educativa.

Otros elementos que pueden favorecer o restringir la realización de las experiencias son los recursos económicos, humanos y edilicios con los que cuentan las instituciones. Algunas experiencias son posibles porque la institución cuenta con recursos propios que se han volcado hacia ciertas actividades o porque una organización externa brinda el soporte material. En situaciones de restricción de recursos entran en juego la voluntad y la creatividad de los participantes, que a pesar de situaciones desfavorables o con limitaciones logran el desarrollo de experiencias significativas a nivel institucional.

El marco institucional del desarrollo de experiencias está dado por series de normativas, materiales de trabajo para los docentes y capacitaciones en los que se llama al desarrollo de las mismas. En la provincia de Santa Fe se 
Revista de la Escuela de Ciencias de la Educación, año 13, nRo. 12, vol. 1, enero a junio de 2017. PáglNAS 175-196. ISSN 1851-6297 - ISSN 2362-3349 (EN LINEA) DIFERENCIACIÓN ESCOLAR Y EXPERIENCIAS PEdagógicas en espacios locales: el caso de las escuelas secundarias de Rosario. Jorgelina Silvia Sassera

dicta un postítulo sobre "Especialización en Proyectos con Jóvenes" que plantea a la escuela

Como plataforma hacia nuevas experiencias de enseñanza aprendizaje extramuros, que ofrezcan a nuestros jóvenes otros vínculos con la realidad social (territorial y virtual), proyectándolos de más y mejores herramientas para tomar distancia, desnaturalizar lo cotidiano y operar de modo transformador y protagónico (Res. 1184/09. p. 2).

La escuela es el lugar de resignificación de la práctica pedagógica mediante la generación de experiencias que propicien nuevos saberes. En este discurso se produce una vinculación entre el conocimiento y el saber-hacer con "el flujo de la vida". Por este motivo el documento plantea una relación entre teoría y práctica no dicotómica pues la reflexión y la acción se imbrican:

Hacer realidad la enseñanza desde la praxis, habilitando un nuevo modelo educativo que impacte también sobre los saberes curriculares a partir de transformar al alumno, al docente y al directivo. Donde el uso de múltiples lenguajes como el de las tecnologías, el arte, el juego y el trabajo, hagan de la acción con otros y para otros la forma de producir conocimiento, autoconciencia y pertenencia a un colectivo (Res. 1184/09. p. 3).

En este apartado se propone una aproximación a la comprensión de la relación entre circuitos educativos y experiencias pedagógicas, buscando indagar la forma en que la diferenciación entre escuelas y otros factores pueden potenciar o limitar el desarrollo de prácticas pedagógicas específicas significativas para los estudiantes.

La aproximación a esta relación, busca dar con una nueva evidencia de formas de segmentación educativa y del conocimiento. En la perspectiva de este artículo, la noción de segmentación da cuenta de la existencia de circuitos, caminos o grupos de escuelas al interior del nivel estudiado que promueven trayectorias educativas desiguales al segregar a los grupos de poblaciones que tienden a homogeneizarse al interior de cada grupo. Siguiendo la línea de lo expresado anteriormente, los circuitos o grupos no se expresan de manera dicotómica, sino en gradientes de diferencias según diversos factores de diferenciación y la localización espacial de las instituciones en territorios heterogéneos y fragmentados.

Las brechas entre grupos de escuelas son tributarias de muchos factores: los recursos materiales, financieros, edilicios y docentes y las relaciones y vinculaciones con actores y organizaciones de la realidad social y económica. Si bien todos estos elementos inciden en la labor pedagógica, también podría indagarse de qué manera determinadas prácticas pedagógicas pueden seguir alimentando a la segmentación educativa. 
Revista de la Escuela de Ciencias de la Educación, año 13, nro. 12, vol. 1, enero a junio de 2017. PáglNAS 175-196. ISSN 1851-6297 - ISSN 2362-3349 (EN LiNEA) DIFERENCIACIÓN ESCOLAR Y EXPERIENCIAS PEdagógicas en espacios locales: el caso de las escuelas secundarias de Rosario. Jorgelina Silvia Sassera

Cuadro 3. Rosario. Tipos de experiencias pedagógicas según grupos de escuelas y adaptaciones curriculares.

\begin{tabular}{|c|c|c|}
\hline Grupo & Orientaciones & Tipos de Experiencias \\
\hline $\begin{array}{l}\text { Grupo I } \\
\text { E.E.T. L } \\
\text { E.E.T P } \\
\text { E.E.T O } \\
\text { E.E.M. C }\end{array}$ & $\begin{array}{l}\text { Maestro Mayor de Obra. } \\
\text { Informática personal y profe- } \\
\text { sional. } \\
\text { Técnico Mecánico de } 3^{\circ} \\
\text { Electromecánica. } \\
\text { Ciencias Sociales. } \\
\text { Ciencias Naturales, Econo- } \\
\text { mía. } \\
\text { Informática personal y profe- } \\
\text { sional. } \\
\text { Industria de Procesos. } \\
\text { Comunicación Multimedial } \\
\text { Arte. } \\
\text { Administración y Gestión } \\
\text { Comunicación. }\end{array}$ & $\begin{array}{l}\text { Orientadas a la formación ciudadana y la inte- } \\
\text { gración social: } \\
\text { - Proyecto socio-comunitario. } \\
\text { - La escuela va a la escuela. } \\
\text { - Mural. } \\
\text { - Juegos integradores. } \\
\text { Orientadas al mundo del trabajo: } \\
\text { - Visita a empresas. } \\
\text { - Pasantía. } \\
\text { Orientadas al fortalecimiento del aprendizaje: } \\
\text { - Convenio con instituto de inglés. }\end{array}$ \\
\hline $\begin{array}{l}\text { Grupo II } \\
\text { E.E.T K } \\
\text { E.E.M B } \\
\text { EET M } \\
\text { E.E.T H } \\
\text { E.E.T. I } \\
\text { EESO D } \\
\text { EESO } \\
\text { NEESO D }\end{array}$ & $\begin{array}{l}\text { Informática, profesional y } \\
\text { personal. } \\
\text { Administración y gestión. } \\
\text { Electrónica Electromecánica. } \\
\text { Comunicación multimedial. } \\
\text { Humanidades y Ciencias } \\
\text { Sociales. } \\
\text { Economía y Administración. } \\
\text { Automotores. } \\
\text { Ciencias Naturales. } \\
\text { Economía y Administración. }\end{array}$ & $\begin{array}{l}\text { Orientadas al mundo del trabajo: } \\
\text { - Prodibur. } \\
\text { - Visita empresas. } \\
\text { - Conexión con agrotécnica. } \\
\text { - Charlas automotriz. } \\
\text { Orientadas al fortalecimiento del aprendizaje: } \\
\text { - La huerta va a la escuela. } \\
\text { - Con el reciclado los chicos aprenden y los } \\
\text { niños juegan. } \\
\text { Orientadas a la formación ciudadana y la inclu- } \\
\text { sión social: } \\
\text { - Visitas al hospital, Grupo Puente. } \\
\text { - Consejal por un día. } \\
\text { - Proyecto socio-comunitario. }\end{array}$ \\
\hline $\begin{array}{l}\text { Grupo III } \\
\text { E.E.M E } \\
\text { E.E.M F } \\
\text { E.E.T. R } \\
\text { E.E.T P } \\
\text { E.E.T. Q } \\
\text { E.E.T T } \\
\text { E.E.T S } \\
\text { E.E.T U }\end{array}$ & $\begin{array}{l}\text { Comunicación, Arte y Diseño. } \\
\text { Economía y administración. } \\
\text { Informática personal y profe- } \\
\text { sional. Gestión y Economía. } \\
\text { Electromecánica. } \\
\text { Electrónica. } \\
\text { Mecánica. } \\
\text { Informática. } \\
\text { Turismo. } \\
\text { Bellas Artes. } \\
\text { Diseño y producción de joyas. } \\
\text { Electromecánica. }\end{array}$ & $\begin{array}{l}\text { Orientadas a la formación ciudadana y la inclu- } \\
\text { sión social: } \\
\text { - Talleres de salud. } \\
\text { - Taller charla de género. } \\
\text { - Creación logo de la escuela. } \\
\text { Orientadas al mundo del trabajo: } \\
\text { - Pasantía con universidad nacional. } \\
\text { - Prácticas profesionalizantes. } \\
\text { - Curso de soldadura. } \\
\text { - Visitas a empresas. }\end{array}$ \\
\hline
\end{tabular}

Fuente: Elaboración propia. PEET-IICE-FFyL/UBA 
Revista de la Escuela de Ciencias de la Educación, año 13, nRo. 12, vol. 1, enero a junio de 2017. PáglNAS 175-196. ISSN 1851-6297 - ISSN 2362-3349 (EN LINEA) DIFERENCIACIÓN ESCOLAR Y EXPERIENCIAS PEdagógicas en espacios locales: el caso de las escuelas secundarias de Rosario. Jorgelina Silvia Sassera

En Rosario, las escuelas del circuito o grupo I, con buenas condiciones edilicias y de recursos localizadas en el distrito centro realizan experiencias pedagógicas orientadas principalmente a la formación ciudadana y al fortalecimiento del aprendizaje; aunque en una institución se realiza una experiencia orientada al mundo del trabajo. Si bien el grupo se compone tanto por escuelas técnicas como por escuelas secundarias comunes, las experiencias no parecen relacionarse directamente con la modalidad ni con la orientación, sino que el surgimiento o necesidad a la que buscan responder, como las organizaciones externas a la escuela que intervienen, son los factores por los que se realizan.

Una de las experiencias de fortalecimiento de los aprendizajes consiste en el otorgamiento de becas de estudio por iniciativa de un instituto privado de inglés. La beca cubre dos años de estudio y los materiales, como libros de texto y cuadernillos. Si bien el propósito es que los estudiantes puedan profundizar sus conocimientos en idiomas y conocer otras culturas, el acceso a esta oportunidad es selectivo, pues las becas son "algunas" y a las mismas acceden los estudiantes con mejor promedio y "conducta". Esta misma institución desarrolla también otra experiencia que consiste en un proyecto solidario en la que se realizan obras de teatro en escuelas primarias con carencias a las que concurren chicos "de la periferia". Esta experiencia tiene la particularidad de que los materiales, el armado de escenografía la realizan los adultos, así como que las ideas también parten de los docentes y los estudiantes realizan la actividad, lo cual da cuenta de relaciones jerárquicas entre adultos y jóvenes. Se espera que de esta experiencia, los estudiantes mejoren su lenguaje apliquen contenidos de materias, desarrollen empatía, respeten a los demás y conozcan otras realidades.

Otra de las experiencias, consiste en viajes didácticos en los que se buscan aplicar conocimientos de historia y geografía, aunque también tienen un componente recreativo. El propósito es que los estudiantes conozcan lugares a los cuales no accederían sin la escuela, y fomentar el compañerismo, el respeto por el espacio público, la motivación y la generación de vínculos entre los estudiantes y los docentes. En este caso, participarían todos los estudiantes del último año, salvo aquellos que posean amonestaciones y se señala que el viaje contribuye, por estos motivos, a la disciplina.

Una experiencia orientada al mundo del trabajo se realiza en una de las escuelas técnicas. Consiste en una pasantía (así se refiere a la misma el docente) con la facultad de agronomía de una universidad pública y surgió por la oportunidad abierta por dos docentes de la escuela que también trabajaban en la universidad. Las actividades que realizaron los estudiantes consistieron en tareas de investigación, lectura y registro de materiales bibliográficos y la experiencia culminó con la realización de una monografía con defensa oral. Esta experiencia parecería no aplicar mecanismos selectivos, pues participarían todos los estudiantes del quinto año. 
Revista de la Escuela de Ciencias de la Educación, año 13, nRo. 12, vol. 1, enero a junio de 2017. PáglNAS 175-196. ISSN 1851-6297 - ISSN 2362-3349 (EN LiNEA) DIFERENCIACIÓN ESCOLAR Y EXPERIENCIAS PEdagógicas en espacios locales: el caso de las escuelas secundarias de Rosario. Jorgelina Silvia Sassera

Además de aprender los temas sobre los que se investiga, se esperó que los estudiantes adquieran habilidades tales como dialogar, transmitir información, interpretar al otro y prestar atención. Es interesante la enumeración de los valores que se espera que los estudiantes adquieran, tales como el respeto a la autoridad, la responsabilidad en el cumplimiento del trabajo y el esmero. En este sentido, coinciden las expectativas sobre los cambios que deberían operarse en la subjetividad de los estudiantes mencionadas respecto a ámbitos laborales privados y coinciden los enfoques actitudinales propios de la meritocracia y del eje puesto en el esfuerzo orientado a objetivos.

En cuanto a las condiciones materiales y relacionales, en el desarrollo de las experiencias, existen vinculaciones con organizaciones y actores de la realidad social y productiva: un instituto de inglés, escuelas primarias, una universidad, las cuales son cultivadas por las escuelas haciendo uso del propio capital social del que disponen por ejemplo, los propios docentes. No existen restricciones en cuanto a posibles recursos necesarios para el desarrollo de las actividades, por ejemplo en el caso de los viajes los estudiantes pagan cuotas para solventar los mismos o en la realización de las obras de teatro los materiales utilizados son dados por la propia escuela.

Las escuelas del circuito o grupo II realizan diversidad de experiencias, principalmente orientadas al mundo del trabajo y a la formación ciudadana y la inclusión social. En este grupo es frecuente que las instituciones desarrollen más de una experiencia que incluso pueden ser de distinto tipo. Esto da cuenta por un lado de que las instituciones no siguen solamente un propósito en la formación de los estudiantes y que la capacidad de estas instituciones para la gestión de recursos (materiales y financieros, aunque también humanos) y de una acumulación de un capital social o relacional que luego aprovechan para el desarrollo de actividades específicas y para una mayor captación de recursos.

En un caso, una escuela técnica con orientación automotriz desarrolla dos tipos de experiencias. En primera instancia charlas con especialistas de empresas automotrices que presentan el funcionamiento de equipamiento específico. Los objetivos de esta práctica son por un lado despertar el interés del alumno, y por el otro ver la aplicación práctica de lo aprendido en clase; también el acercamiento a tecnología de última generación "que no se ve en los libros". Además del conocimiento técnico implicado, se espera que los estudiantes adquieran "amor por el trabajo", respeto, y que sigan el ejemplo del instructor que dicta la charla. Participan todos los estudiantes del sexto año, y por este motivo parecerían no existir mecanismos selectivos explícitos.

La segunda experiencia se orienta a la formación ciudadana y la inclusión social, aunque podría tener también componentes de fortalecimiento del aprendizaje. Esto da cuenta de las limitaciones de la tipología de experiencias, pues según los atributos considerados, la experiencia podría clasificarse en uno o en otro tipo. La actividad en cuestión consiste en la fabricación de ju- 
Revista de la Escuela de Ciencias de la Educación, año 13, nro. 12, vol. 1, enero a junio de 2017. PáglNAS 175-196. ISSN 1851-6297 - ISSN 2362-3349 (EN LINEA) DIFERENCIACIÓN ESCOLAR Y EXPERIENCIAS PEdagógicas en espacios locales: el caso de las escuelas secundarias de Rosario. Jorgelina Silvia Sassera

guetes y de mobiliario infantil realizados con maderas recicladas en el taller de carpintería, y que luego son donados al hospital de niños. La experiencia surge en virtud de la relación del personal directivo de la escuela con la dirección del hospital y del impulso recibido mediante un programa municipal que fomenta el reciclaje. En este caso, participan estudiantes de varias divisiones de la escuela, con énfasis en estudiantes repitentes con la finalidad de "motivarlos", pues en la realización práctica de los productos, se ponen en juego conocimientos de matemática y tecnología con la manipulación de herramientas y la aplicación de técnicas como el lustre.

En otras instituciones, existe una gran diversidad de experiencias orientadas al mundo del trabajo, desde la conexión con una escuela agrotécnica mediante la cual los estudiantes contribuyeron al diseño de una incubadora haciéndose cargo de la electrónica hasta visitas a empresas alimenticias que consistieron en el recorrido de la planta. Otras experiencias, se refieren a la formación ciudadana, como la simulación de práctica parlamentaria en al cual los estudiantes deben desarrollar un proyecto y luego presentarlo para discutir ante el Consejo Deliberante local.

Una de las secundarias comunes también desarrolla dos tipos de experiencias, en este caso orientadas al mundo del trabajo y al fortalecimiento del aprendizaje. La primera se sustenta en un dispositivo informático de simulación y participación en un certamen contable organizado por la Bolsa de Comercio de Buenos Aires y del que participa la Bolsa de Comercio de Rosario, y se espera que la participación sea obligatoria para los estudiantes del último año pues quienes no desean participar deben compensar con un trabajo práctico. La otra experiencia consistió en la creación de una huerta en la escuela con la finalidad de "despertar" el interés de los estudiantes en la materia de Biología, esto se desarrolló por propia iniciativa de la institución que brinda semillas y herramientas a los estudiantes.

En el grupo III de escuelas, hay una institución que no desarrolla ningún tipo de experiencia pedagógica, pero mantiene vínculos con diversas organizaciones y actores de la realidad social y productiva. Por ejemplo, con clubes del barrio para que los estudiantes asistan a hacer deportes pues la escuela no cuenta con el espacio requerido para ello; y con la Municipalidad de la cual reciben invitaciones para asistir a obras de teatro. Sin embargo, se mencionan limitaciones en estos vínculos, debido especialmente a la localización periférica del sur.

Otras instituciones realizan experiencias orientadas al mundo del trabajo y a la formación ciudadana y la inclusión social. Es preciso recordar, que las instituciones de este grupo, cuentan con menores recursos y con condiciones edilicias menos favorables que las escuelas de los circuitos o grupos I y II; en estas instituciones además, se concentraría la matrícula estudiantil con mayores dificultades socio - económicas. 
Revista de la Escuela de Ciencias de la Educación, año 13, nRo. 12, vol. 1, enero a junio de 2017. PáglNAS 175-196. ISSN 1851-6297 - ISSN 2362-3349 (EN LiNEA) DIFERENCIACIÓN ESCOLAR Y EXPERIENCIAS PEdagógicas en espacios locales: el caso de las escuelas secundarias de Rosario. Jorgelina Silvia Sassera

Por ejemplo en una de las escuelas se desarrollan talleres sobre la violencia de género, organizados por la Municipalidad; luego de las actividades dispuestas por los talleristas, los estudiantes realizaron un mural sobre tema que demandó dos meses de trabajo con materiales dados por el municipio. Una experiencia similar se desarrolló en otra escuela, en la cual profesionales de la salud de una universidad pública establecieron una consejería sobre temas de salud reproductiva y sexualidad, también talleres sobre violencia y maltrato. En otra institución se impulsó a que todos los estudiantes diseñen un logo para la escuela, el cual luego fue votado por los vecinos del barrio. La experiencia tuvo el doble propósito de que los adolescentes apliquen técnicas de dibujo y aprendan la utilización de programas de computación.

En algunas de las instituciones se desarrollan experiencias orientadas al mundo del trabajo. En un caso consistieron en visitas a empresas del sector de alimentos. Participan todos los estudiantes del ciclo superior y se realizan desde el área contable con dos finalidades: que puedan ver aplicados los conocimientos de las materias, llevar a la práctica lo enseñado; y que puedan conocer otras zonas de Rosario pues

La mayoría son chicos carenciados que no tienen idea qué es una fábrica, tienen que verla. La única vida extra familiar es la escuela [...] Porque los chicos no salen de su barrio, de su villa, no conocen el trato, como comportarse (Docente, Rosario).

En este sentido, se sostiene que la escuela "es un ensayo para trabajar" pues se señala que serán pocos los estudiantes de la escuela que seguirán estudiando y se da por hecho en cierto sentido que "la mayoría va a trabajar". Por esto, se espera que de la visitas, los estudiantes aprendan lo que es la responsabilidad del trabajo, el cumplimiento de normas, horarios y el uso de uniforme.

En otra institución, que presenta situaciones de desfavorabilidad, dificultades de accesibilidad y abandono escolar, se brindaron cursos de soldadura. El instructor demostró las técnicas y equipamiento y luego los estudiantes tuvieron la posibilidad de hacer prácticas. La finalidad de esta experiencia fue brindar los conocimientos necesarios favorecer la inserción laboral de estos estudiantes en un "taller pequeño". La capacitación se realizó durante varios días en los que rotaron grupos de estudiantes desde $3^{\circ}$ hasta $6^{\circ}$ año; incluso participaron docentes y preceptores. Los equipos fueron brindados por un ex alumno de la institución que fue el instructor y promotor de la experiencia y se solventaron los gastos de materiales utilizando recursos del Plan de Mejoras Institucional.

Un interrogante en el análisis apunta a si estas experiencias pedagógicas pueden actuar como un factor de diferenciación, especialmente cuando existen prácticas selectivas. En algunas experiencias, los docentes eligen según 
Revista de la Escuela de Ciencias de la Educación, año 13, nro. 12, vol. 1, enero a junio de 2017. PáglNAS 175-196. ISSN 1851-6297 - ISSN 2362-3349 (EN LINEA) DIFERENCIACIÓN ESCOLAR Y EXPERIENCIAS PEdagógicas en espacios locales: el caso de las escuelas secundarias de Rosario. Jorgelina Silvia Sassera

criterios meritocráticos a los estudiantes que participan, desde las calificaciones del "buen estudiante" hasta la evaluación de las actitudes tales como el interés o la demostración de compromiso. Otras experiencias serían formalmente no selectivas, pero la "participación" de los estudiantes queda delimitada por la voluntad no explicitada del docente. En contrapartida en algunas instituciones se busca la inclusividad, la mayor participación posible de los estudiantes e incluso de los docentes pues se considera que esto redunda en un beneficio colectivo para la institución.

Un punto que muestra relación entre las diferencias entre las instituciones (y la matrícula que la componen) y los tipos de experiencias refiere a la dotación de recursos y la capacidad de gestión. Por ejemplo, aquellas escuelas que desarrollan lecciones paseo y excursiones dan cuenta de una capacidad de gestión administrativa (pues consiste en un dispositivo muy burocrático) y da cuenta también de la disponibilidad de recursos monetarios, ya sea porque pueden pagarlo los estudiantes o la institución gestiona recursos por otros medios.

Si bien la orientación de la escuela no es determinante en el tipo de experiencia pedagógica, en las escuelas técnicas, suelen predominar las experiencias orientadas al mundo del trabajo. Podría deberse a que ciertos perfiles institucionales son los más "elegidos" por las empresas y otras organizaciones para el desarrollo de estas experiencias y en este sentido predomina una noción mercantilizada del trabajo, según la cual los estudiantes deben ser atractivos para el mercado laboral, no sólo en cuanto a los conocimientos, sino en su forma de ser. Se espera que sean respetuosos, atentos a las jerarquías, sigan las reglas y trabajen en equipo. Esto contrasta con una noción del trabajo subyacente en las experiencias de formación ciudadana e inclusión social, en las cuales el trabajo adopta la forma de la creatividad, el compañerismo y la solidaridad. En estas experiencias, la realización de un trabajo significa unir a los sujetos y crear lazos entre ellos y con la institución.

\section{A modo de cierre}

Este artículo abordó procesos de diferenciación institucional en un espacio local concreto, la ciudad de Rosario y tomó como un eje de acercamiento a este problema la realización de experiencias pedagógicas en escuelas secundarias. Se ha analizado de manera relacional las características de las escuelas con su localización en el espacio. La investigación muestra que si bien no existen situaciones polarizantes o quiebres insalvables entre las realidades institucionales, se registran gradientes de diferencias que pueden significar accesos diferentes y diferenciantes a la educación por parte de los adolescentes.

El tratamiento de la diferenciación y de la segmentación educativa muestra que las brechas entre grupos de escuelas son tributarias de múltiples factores: los recursos materiales, financieros, edilicios y docentes y las relaciones y 
Revista de la Escuela de Ciencias de la Educación, año 13, nRo. 12, vol. 1, enero a junio de 2017. PáglNAS 175-196. ISSN 1851-6297 - ISSN 2362-3349 (EN LiNEA) DIFERENCIACIÓN ESCOLAR Y EXPERIENCIAS PEdagógicas en espacios locales: el CASO de las escuelas seCundarias de Rosario. Jorgelina Silvia Sassera

vinculaciones con actores y organizaciones de la realidad social y económica. Si bien todos estos elementos inciden en la labor pedagógica, también podría indagarse de qué manera determinadas experiencias pedagógicas pueden seguir alimentando a la segmentación educativa.

En este artículo, se abordaron las relaciones entre circuitos o grupos de escuelas en Rosario con la realización de experiencias pedagógicas, para mostrar cómo la desigualdad surgida de la diferenciación institucional puede afectar ciertas prácticas de la enseñanza y el aprendizaje. La investigación que da origen a este artículo mostró que existen diferencias entre los tipos en relación con la dotación de recursos y capacidad de gestión. Por ejemplo, aquellas escuelas que desarrollan lecciones-paseo o excursiones dan cuenta de una capacidad de gestión administrativa (pues consiste en un dispositivo muy burocrático) y también de la disponibilidad de recursos monetarios.

La aproximación a las experiencias pedagógicas, en el marco de críticas realizadas hacia el aprendizaje "tradicional" o mediante sólo "los libros" es posible gracias a la participación de diversos actores en el marco de la legitimidad construida por la normativa escolar que alude a que los aprendizajes realizados por este medio redundarían no solamente en la adquisición de determinados conocimientos y habilidades, sino en cambios subjetivos y actitudinales en los estudiantes. En esta instancia podría plantearse que en las experiencias pedagógicas se produce un cruce y una tensión entre la transmisión de lo que Michael Young denominó conocimiento poderoso con un conocimiento contextual y vivencial, en paralelo con formas de saber hacer que limitan con un saber - ser del sujeto.

Cabe marcar, sin embargo, que no puede plantarse una relación lineal entre localización de las escuelas y los tipos de experiencia que desarrollan, pues afecta de manera distinta a las escuelas estudiadas. Por otra parte, al tratarse de una muestra de instituciones, debe tenerse precaución en la generalización de los resultados de la investigación.

Queda presentada una línea de investigación que considera que la diferenciación en las posibilidades de aprendizajes de los adolescentes ocurre en espacios concretos, según circuitos educativos en los que no tienen que ver solamente las condiciones materiales de las instituciones, sino también las localizaciones y apropiaciones de recursos espaciales desiguales, los docentes, las capacidades de gestión del equipo directivo, las relaciones con distintos actores y dimensiones pedagógicas específicas ejemplificadas mediante las experiencias pedagógicas. 
Revista de la Escuela de Ciencias de la Educación, año 13, nro. 12, vol. 1, enero a junio de 2017. PáglNAS 175-196. ISSN 1851-6297 - ISSN 2362-3349 (EN LINEA) DIFERENCIACIÓN ESCOLAR Y EXPERIENCIAS PEdagógicas en espacios locales: el caso de las escuelas secundarias de Rosario. Jorgelina Silvia Sassera

\section{Notas bibliográficas}

1) Este artículo es resultado de los proyectos de investigación "Educación y formación para el trabajo. Deuda social con jóvenes y adultos y mercado de ilusiones de corto plazo" (UBACyT 2011-2014 y PIP CONICET) y Proyecto "Los escenarios complejos de la educación y formación para el trabajo: vigencia, nuevos sentidos y el papel de los actores sociales. (UBACyT Programación Científica 2014 -2017 GC), dirigidos por la Dra. Graciela C. Riquelme con sede en el Programa Educación, Economía y Trabajo, Instituto de Investigaciones en Ciencias de la Educación, Facultad de Filosofía y Letras, Universidad de Buenos Aires. En el marco de estos proyectos se realizó la tesis doctoral "Educación y realidad social y productiva en ámbitos locales: el sentido de las experiencias pedagógicas de adolescentes y jóvenes" (dirigida por la Dra. Graciela C. Riquelme). La investigación se realizó a partir de 2013 hasta la actualidad y consta de una muestra intencional de escuelas secundarias (también de centros de formación profesional y escuelas de jóvenes y adultos) que ha buscado acceder a la mayor cantidad posible con la finalidad de obtener mayor variabilidad, riqueza y diversidad en los datos; así como mayor validez de los mismos. Este artículo presenta un análisis enfocado en las escuelas secundarias de Rosario, esta muestra específica estuvo compuesta por nueve escuelas secundarias comunes y trece escuelas técnicas. Se analizaron diversos documentos y se realizaron entrevistas semi-estructuradas a directivos, docentes y estudiantes.

2) La noción de práctica pedagógica- desarrollada por Bernstein (1996) consta de dos caras, por un lado la del contenido- lo que se transmite, y la de transmisor cultural o sea la forma social mediante se transmite el contenido. La forma de la transmisión de los contenidos se basa en reglas de orden regulador y reglas de orden discursivo que pueden dar lugar a modalidades de las prácticas.

3) Según datos del Censo 2010 , cerca del $27 \%$ los hogares no tienen acceso a las redes de gas o de cloaca; y el 6,02\% presentan al menos una condición de Necesidades Básicas Insatisfechas (NBI). Si se considera la distribución espacial de los hogares, el centro rosarino cuenta con la menor proporción de estos hogares, mientras que existen concentraciones en el sur y el noroeste de la ciudad que alcanzan el $58 \%$.

\section{Referencias Bibliográficas}

- Bernstein, B. (1996). "Introducción”. En Bernstein, B. Pedagogía, control simbólico e identidad. Barcelona: Morata.

- Braslavsky, C. (1994). "Transformaciones en curso en el sistema educativo argentino". En Puryear J. y J. Brunner. (eds). Educación, equidad y competitividad 
Revista de la Escuela de Ciencias de la Educación, año 13, nRo. 12, vol. 1, enero a junio de 2017. PáglNAS 175-196. ISSN 1851-6297 - ISSN 2362-3349 (EN LiNEA) DIFERENCIACIÓN ESCOLAR Y EXPERIENCIAS PEdagógicas en espacios locales: el caso de las escuelas secundarias de Rosario. Jorgelina Silvia Sassera

económica en las Américas, vol. II, Estudios de Caso, Portal educativo de las Américas. Consultado el 3 de mayo de 2013, http://www.educoas.org/Portal/ bdigital/contenido/interamer/BkIACD/Interamer/puryear40.htm

- Dubet, F. (2011). La experiencia sociológica. Barcelona: Gedisa.

- Herger, N. y J. Sassera. (2015). "Educación y formación para el trabajo en Campana, Zárate y Rosario. Diferenciación institucional y mercado de ilusiones" en $12^{\circ}$ Congreso Nacional de Estudios del Trabajo "El trabajo en su laberinto. Viejos y nuevos desafíos", organizado por la Asociación Argentina de Especialistas en Estudios del Trabajo, 6 al 7 de agosto de 2015, Facultad de Ciencias económicas UBA. http://www.aset.org.ar/2015/ponencias/12_Herger.pdf

- Herzer, H. (2005). Situación del hábitat de los municipios del área metropolitana de Rosario en materia de suelo y vivienda, Documento de Proyectos. Santiago de Chile: CEPAL.

- Riquelme, G. C. (1989). Educación y trabajo en zonas desfavorables. Reconceptualización y alternativas en la perspectiva de las políticas sociales. Versión preliminar. Buenos Aires: Ministerio de Educación y Justicia, Organización de los Estados Iberoamericanos.

- Sassera, J. (2014). Tesis de doctorado "Educación y realidad social y productiva en ámbitos locales: el sentido de las experiencias pedagógicas de adolescentes y jóvenes". Directora: Dra. Graciela C. Riquelme. Universidad de Buenos Aires, Facultad de Filosofía y Letras.

- Sassera, J. (2013a). Tesis de maestría "Educación, sociedad y trabajo en la ciudad de Campana: acceso de los adolescentes y jóvenes a la educación y formación para el trabajo". Directora: Dra. Graciela C. Riquelme. Facultad Latinoamericana de Ciencias Sociales, (FLACSO-Argentina).

- Sassera, J. (2013b). "La transición hacia la secundaria obligatoria de seis años en la provincia de Buenos Aires: diferenciación institucional en la ciudad de Campana", en Revista del IICE, $\mathrm{n}^{\circ}$ 33, Buenos Aires, Instituto de Investigaciones en Ciencias de la Educación,FFyL-UBA. http://revistascientificas.filo.uba.ar/index.php/iice/ article/view/1101

- Veleda, C. (2012). La segregación educativa. Entre la fragmentación de las clases medias y la regulación atomizada. Buenos Aires: Editorial Stella, La Crujía.

\section{Normativa citada}

- Resolución CFE No 93/09. Orientaciones para la organización pedagógica e institucional de la educación secundaria obligatoria. Anexo. № 4 (impreso).

- Resolución No 1184/09. Provincia de Santa Fe, Ministerio de Educación. "Proyecto de Postítulo de Especialización Superior en Proyectos Estratégicos con Jóvenes”. 
- 